\title{
Building crack monitoring based on digital image processing
}

\author{
Yanyan Xu, Yanxia Cai*, Dandan Li,Tierui Zhang \\ Hengshui University, Hengshui, Hebei Province 053000, China \\ xyy_xu@126.com,yxcyanx@yeah.net,rzaod5@163.com
}

\begin{abstract}
Building crack monitoring is of great value to the judgment of building safety. In this study, the digital image processing technology was studied and applied to the monitoring of building cracks. Crack images were collected by CCD camera, and then operations such as graying, correction, denoising and segmentation were carried out to obtain clear crack images. The obtained images were processed morphologically to further improve the quality. Finally, the width and length of cracks were calculated. In the case analysis, the results of 15 cracks measured by a microscope were taken as the standards and compared with the calculated results. The results showed that the results calculated in this study and the manual measurement results differed little, and the average errors of the width and length were $0.021 \mathrm{~mm}$ and $0.024 \mathrm{~mm}$ respectively, which suggested that the method proposed had a high reliability. The findings of this study provide a new idea for the further development of the building crack monitoring field and is conducive to the accurate assessment of building safety.
\end{abstract}

KEYWORDS. Digital image processing; Building crack; Monitoring; Histogram equalization.

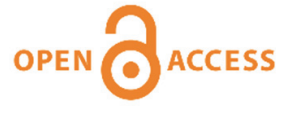

Citation: Xu, Y.Y., Cai, Y.X., Li, D.D., Zhang, T.R., Building crack monitoring based on digital image processing, Frattura ed Integrità Strutturale, 52(2020) 1-8.

Received: 06.08.2019

Accepted: 05.12.2019

Published: 01.04.2020

Copyright: (C) 2020 This is an open access article under the terms of the CC-BY 4.0, which permits unrestricted use, distribution, and reproduction in any medium, provided the original author and source are credited.

\section{INTRODUCTION}

I $\mathrm{n}$ the process of concrete production, due to the uneven expansion and contraction, cracks will inevitably occur in the ground. In the process of component transportation and assembly, cracks may also occur due to the influence of external environment. In the process of building use, cracks will gradually occur along with the deformation of materials. Building cracks will continue to develop and expand to destroy the integrity of the building structure, resulting in a decline in the mechanical properties of the overall structure; if serious, it may lead to shorter building life and cause safety accidents. Cracks in buildings develop slowly, and changes in the width and length can reflect the safety of buildings. Therefore, effective monitoring of cracks has important practical values [1]. With the development of technology, digital image processing has shown excellent performance in crack monitoring. Riyadi et al. [2] monitored pavement cracks with digital image processing technology, developed detection technology through the Gauss pyramid method, classified cracks and non-cracks by linear discrimination, and found that the method had an accuracy of $92.8571 \%$ and the processing time of each picture was 1.5 seconds. Kim et al. [3] combined Unmanned Aerial Vehicle (UAV) technology with digital image processing technology to realize the crack assessment of concrete structures and found that the system could successfully 
measure cracks with thickness greater than $0.1 \mathrm{~mm}$ and the estimated error of the maximum length was $7.3 \%$. Hoang et al. [4] studied the detection and classification of cracks in asphalt pavement, processed images with digital image processing technology, classified images by machine learning algorithm, and found that the method was helpful to assist inspectors in assessing pavement conditions. Lu et al. [5] studied the cracks of cement matrix composites, designed a double threshold algorithm for image processing, and found through experiments that the proposed method could effectively calculate the crack width. Felli et al. [6] studied related cracks of the right foreleg of Colleoni equestrian statue and made long-term examination on the crack expansion through pasting Fiber Bragg Grating sensor. In this study, a series of digital image processing methods were designed for building cracks, and the example analysis verified that the proposed method had high monitoring accuracy, which provides some bases for its practical application and is conducive to the effective evaluation of building health and the improvement of safety and reliability of buildings.

\section{DIGITAL IMAGE PROCESSING}

$\mathrm{D}$ igital images refer to images composed of data points (pixels). Taking a 360*500 image as an example, it refers to an image which is composed of 360 rows of pixels and 500 columns of pixels. Digital image processing refers to the transformation of image signal to digital signal through computer. Digital image processing has many advantages: (1) high accuracy: by processing each pixel in the image, the image can maintain a high accuracy; (2) high processing speed: data-based digital images can perform various operations quickly in the process of processing; (3) easy storage: unlike paper images, digital images are not affected by time and are easy to store; (4) a wide range of applications: no matter what kind of equipment the image is collected, it can be processed by digital image processing; (5) high flexibility: linear and non-linear processing can be realized.

In the actual acquisition, the image of building cracks may be affected by illumination, photography, occlusion and so on, which makes the boundary between cracks and background unclear and makes it difficult to accurately extract cracks. Clear crack images can be obtained through digital image processing technology to realize the monitoring of cracks.

\section{IMAGE PROCESSING METHOD FOR BUILDING CRACKS}

\section{Image acquisition} $\mathrm{n}$ order to obtain high-quality images, the selected image acquisition equipment should have high pixel and resolution. In this study, MV-VDM200SM/SC CCD industrial camera produced by Video Digital Image Company (Fig. 1) was used. Some of its parameters are shown in Tab. 1.

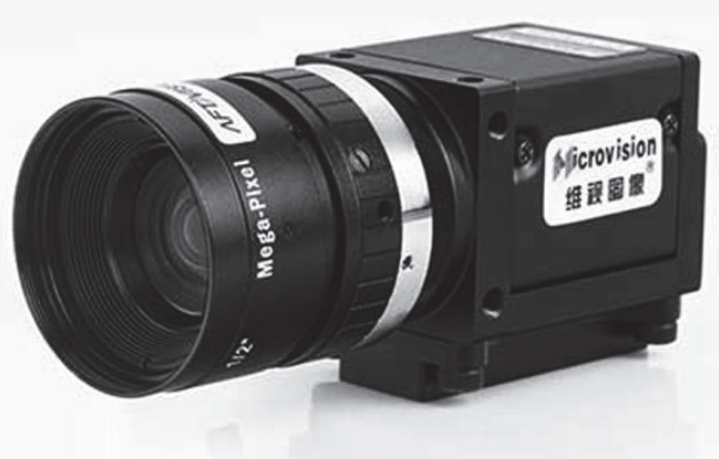

Figure 1: CCD camera.

\begin{tabular}{cc}
\hline Maximum resolution & $1600^{*} 1200$ \\
Pixel size & $4.40 \mu \mathrm{m} \times 4.40 \mu \mathrm{m}$ \\
Frame rate & $12 \mathrm{fps}$ \\
Output mode & $\mathrm{USB} 2.0$ \\
Power supply requirements & $5 \mathrm{~V}$ \\
Power & $2.4 \mathrm{~W}$ \\
\hline
\end{tabular}

Table 1: Parameters of CCD camera. 
In the process of acquisition, the lens was located in front of the crack. The stable image was obtained by tripod and level instrument, and a ruler was set around the crack to provide a standard for crack calculation. Then the acquired image was input to the computer through USB.

\section{Image preprocessing}

Under the influence of illumination and noise, the collected images often have some problems, such as low clarity and inconspicuous details, which are not conducive to crack monitoring. Therefore, image preprocessing is needed to improve the quality.

\section{Image graying}

The image captured by camera is usually color image in RGB format. The amount of calculation involved in the processing is considerable, which can seriously affect the speed of image processing. Therefore, it is necessary to gray the RGB image. In this study, graying was achieved by the weighted average method [7]. It is assumed that there is an RGB image $f(i, j)$. The treatment formula is:

$$
f(i, j)=0.3 R(i, j)+0.59 G(i, j)+0.11 B(i, j) \quad .
$$

Gray level correction

In order to improve the gray resolution of the image, gray correction can be made to the image. In this study, the image was corrected by the histogram equalization method [8].

Firstly, the gray histogram $p\left(r_{k}\right)$ of the image is calculated: $p\left(r_{k}\right)=\frac{n_{k}}{N}, k=0,1, \cdots, 225$, where $r_{k}$ stands for the $k$-th grayscale, $N$ stands for number of pixels, $n_{k}$ stands for the number of pixels with gray level of $k$, and $p\left(r_{k}\right)$ stands for the proportion of $r_{k}$ in the whole image.

The cumulative distribution function $s_{k}$ is calculated:

$$
s_{k}=\sum_{j=0}^{k} \frac{n_{j}}{n} \text {. }
$$

$s_{k}$ is taken as a transform function to correct the gray level of the image: $g_{k}=\frac{s_{k} \times 225}{N}+0.5$, where $g_{k}$ stands for the new grayscale of the corrected image. All the gray levels of the image are corrected according to the above procedures, and then the corrected gray level image is obtained.

Image denoising

The image is denoised by Median filter [9], that is, the gray value in window $W$ is ranked. Then the median value is used as the gray value of the central point. The calculation formula is:

$$
g(p, q)=\operatorname{med}\{f(p-i, q-j),(i, j) \in W\}
$$

where $f(i, j)$ stands for the original gray value in $W$ and $g(p, q)$ stands for the gray value after median filtering denoising.

\section{Image segmentation}

Cracks and background are separated by image binarization. For the original image $f(x, y)$, threshold $T$ is set, and the segmented image is:

$$
g(x, y)=\left\{\begin{array}{l}
1 f(x, y) \geq T \\
0 f(x, y)<T
\end{array} .\right.
$$


The method for determining the threshold is as follows. Firstly the initial threshold $T=\frac{T_{\max }+T_{\min }}{2}$ is calculated, where $T_{\max }$ and $T_{\min }$ stand for the maximum and minimum gray values in the image respectively. The image gray level is divided into two groups, group 1 with gray level larger than $T$ and group 2 with gray level smaller or equal to $T$. The average gray values $\sigma_{1}$ and $\sigma_{2}$ of the two groups are calculated. Suppose $T=\frac{\sigma_{1}+\sigma_{2}}{2}$. The above procedures repeat until $T$ is stable, and finally the binarization threshold is obtained.

\section{CRACK CALCULATion}

\section{Morphological treatment}

7 or the segmented crack image, the morphological algorithm is taken for further improvement, which mainly includes 1 two steps: corrosion and expansion.

(1) Corrosion: $A \Theta B=\{x \mid B(x) \subseteq A\}$, where $A$ stands for the target image and $B$ stands for the structural element. The noise and burr in the image can be further removed after corrosion.

(2) Expansion: $A \oplus B=\{x \mid B(x) \cap x \neq \Phi\}$. After expansion, the image can be restored to the original size.

\section{Calculation of crack width}

Coordinates are set around the crack to calculate the distance between the two points: $w=\eta \sqrt{\left(x_{\text {right }}-x_{\text {left }}\right)^{2}+\left(y_{\text {right }}-y_{\text {left }}\right)^{2}}$, where $\eta$ represents the pixel scale parameter, which is the actual size represented by the pixel. As cracks are mostly irregular, the coordinates of left pixels are kept unchanged in the calculation, and then the coordinates of right pixels are calculated in turn with the window size of $5 \times 5$, and the minimum value is denoted as the crack width.

\section{Calculation of crack length}

The length of crack is calculated through the Euclidean distance between the starting point of crack $\left(x_{s}, y_{s}\right)$ and the ending point $\left(x_{e}, y_{e}\right): l=\eta \sqrt{\left(x_{e}-x_{s}\right)^{2}+\left(y_{e}-y_{s}\right)^{2}}$, where $\eta$ stands for the pixel proportion parameter.

\section{CASE STUDY}

\section{Crack image acquisition}

7 ifteen cracks were collected by CCD camera in building A in Hengshui, Hebei, China. The data of the length and width of the fifteen cracks were collected using a WYSK-40X reading microscope (Fig. 2). The specification of the microscope was $50 \times 23 \times 138 \mathrm{~mm}$. The focus of the microscope was adjustable, and it carried with pure white LED light source, had scale, and had 40X magnification.

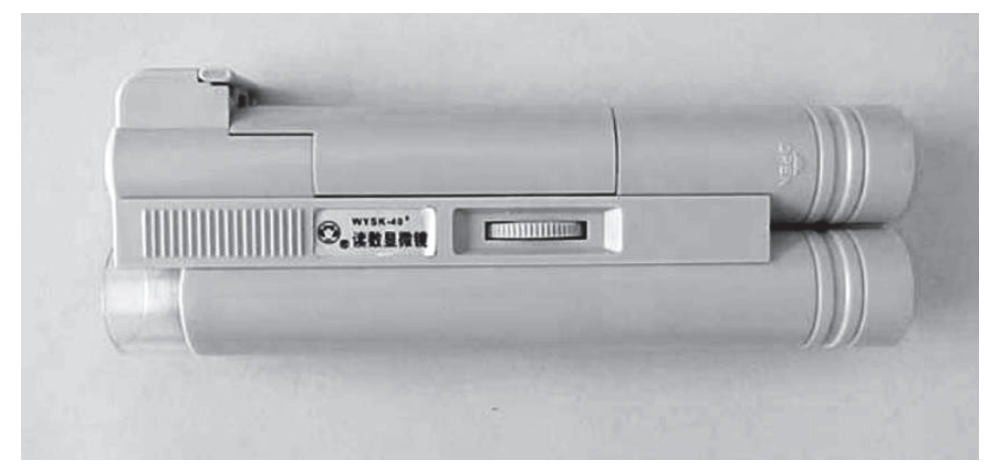

Figure 2: The reading microscope. 
Image processing

The images were processed using the method described in the section of image preprocessing. Four of the images were taken as examples, and the original images of the four images are shown in Fig. 3.

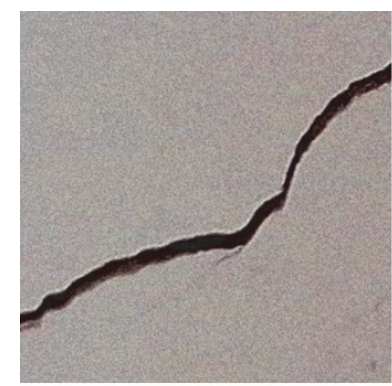

(1)

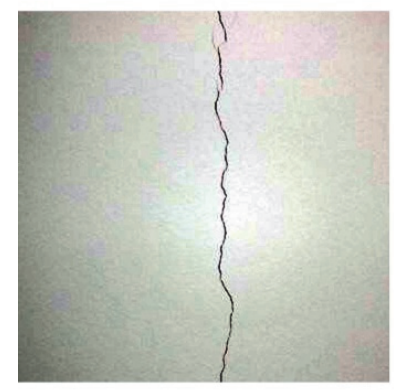

(3)

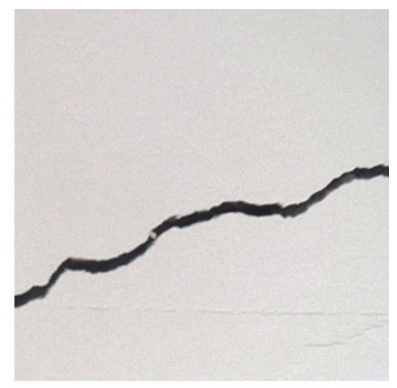

(2)

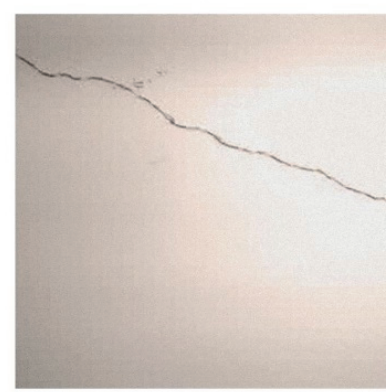

(4)

Figure 3: Original crack images.

It was found from Fig. 3 that the quality of crack images was poor under the influence of illumination and noise, which was not conducive to crack monitoring. The crack images obtained after the processing of the digital image processing method proposed in this study are shown in Fig. 4.

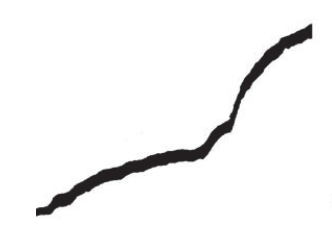

(1)

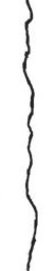

(3)

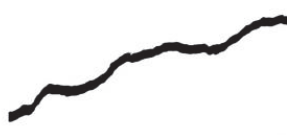

(2)

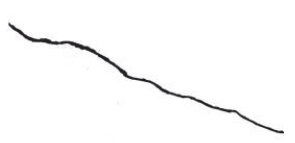

(4)

Figure 4: Crack images after processing.

It was found from Fig. 4 that the crack images obtained were clearer and distinct from the background after processing such as denoising, segmentation and morphological treatment, which was conducive to the subsequent crack calculation.

\section{Crack calculations}

The width and length of the extracted crack images were calculated using the method mentioned in the section of crack calculation. The comparison between the calculated results and manual monitoring results is shown in Fig. 5 and 6. 


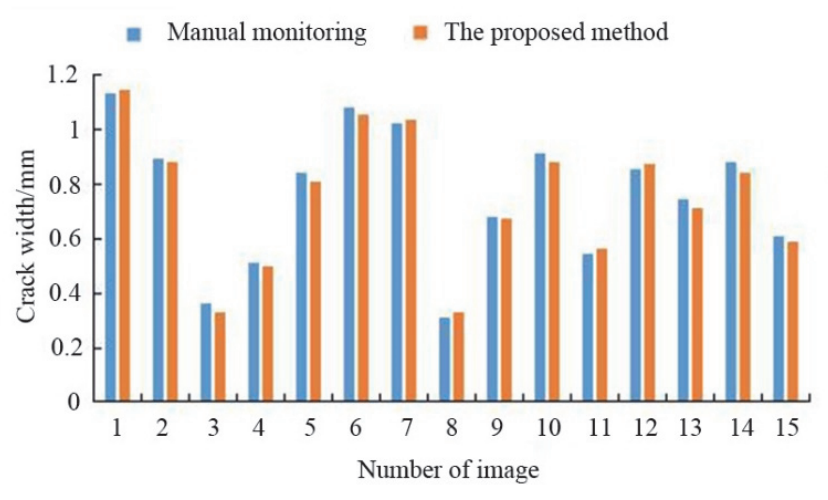

Figure 5: Comparison of crack width.

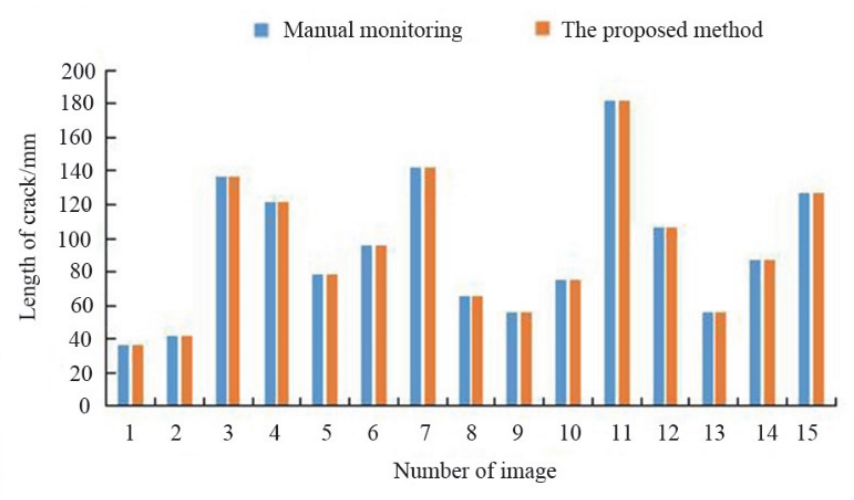

Figure 6: Comparison of crack length.

It was found from Fig. 5 and 6 that the width and length of cracks calculated by the method were almost the same as the measured values, which showed that the method had a high monitoring accuracy and could replace manual monitoring to achieve effective monitoring of cracks.

The errors between the results obtained by the proposed method and those obtained by manual monitoring are shown in Tab. 2.

\begin{tabular}{ccc}
\hline Number of image & Width error $/ \mathrm{mm}$ & Length error $/ \mathrm{mm}$ \\
1 & 0.01 & 0.03 \\
2 & 0.01 & 0.01 \\
3 & 0.03 & 0.02 \\
4 & 0.01 & 0.03 \\
5 & 0.03 & 0.03 \\
6 & 0.03 & 0.04 \\
7 & 0.01 & 0.04 \\
8 & 0.02 & 0.03 \\
9 & 0.01 & 0.01 \\
10 & 0.03 & 0.02 \\
11 & 0.02 & 0.02 \\
12 & 0.02 & 0.02 \\
13 & 0.03 & 0.03 \\
14 & 0.04 & 0.02 \\
15 & 0.02 & 0.01 \\
Average error & 0.021 & 0.024 \\
\hline
\end{tabular}

Table 2: Errors between the results obtained using the method proposed in this study and the manual detection results

It was found from Tab. 2 that the maximum error of the method was $0.04 \mathrm{~mm}$, the minimum error was $0.01 \mathrm{~mm}$, and the average error was $0.021 \mathrm{~mm}$ in the width monitoring; in the length monitoring, the maximum error was $0.04 \mathrm{~mm}$, the minimum error was $0.01 \mathrm{~mm}$, and the average error was $0.024 \mathrm{~mm}$. The errors were so small that could be nearly neglected, which would not affect the evaluation of cracks, suggesting that the method was reliable. All the results showed that the digital image processing method could obtain almost the same results as the manual monitoring, with a good accuracy, and could realize the effective monitoring of building cracks.

\section{Discussion}

igital image processing has a good application in many fields [10]. For example, in the field of medicine, it can analyze ultrasound and electrocardiogram images [11] to provide a guidance for doctors' surgery [12]. In the field of industry, it can realize the analysis and detection of circuits, chips, micro parts, etc [13,14]. In the field of 
security, it can identify fingerprints and iris [15]. In the field of art, it can realize the restoration and reconstruction of cultural relic pictures [16]. Cracks in buildings are closely related to the overall safety of buildings. Monitoring cracks is an important work of building health assessment. Traditional manual monitoring methods have many limitations in practical operation, which cannot meet the current needs of building crack monitoring. The emergence of digital image processing technology has brought a new idea for building crack monitoring.

This study collected the building crack images by CCD camera, then obtained the clear crack images by a series of pretreatment operations such as graying, denoising and segmentation, and finally realized the monitoring of building cracks by taking the width and length as the criteria. The processing method proposed in this study was found effective in the example analysis. It was found from Fig. 4 that the blur, noise and stain in the original images were effectively removed, the image quality was significantly improved, and the cracks were clearly separated from the background, which was conducive to the follow-up operation. Then, in the comparison of the results of crack length and width, the data measured by microscope was taken as the result of manual monitoring and compared with those calculated by the method proposed in this study. Fig. 5 and 6 show that the results obtained by the two methods were very similar, which was also verified in the calculation of errors. The width error was only $0.021 \mathrm{~mm}$, and the length error was only $0.024 \mathrm{~mm}$. With a high accuracy, the method can realize monitoring of the cracks in buildings. In practice, the method not only has high reliability, but also is convenient, fast and highly usable. However, the manual monitoring method based on microscope is very difficult to achieve in the monitoring of a large number of cracks as it is time-consuming and energy consuming. Therefore, the method is more suitable for the monitoring of actual building cracks.

In this study, although some achievements have been made in the research of building crack monitoring, there are still many shortcomings, for example, unable to achieve on-line crack monitoring and the identification of complex cracks. In the future work, it is necessary to find more accurate monitoring methods and monitor more characteristics of cracks such as area and depth.

\section{CONCLUSION}

$\mathrm{I}$

$\mathrm{n}$ this study, the monitoring of building cracks was studied, clear crack images were obtained through digital image processing technology, and the length and width of the cracks were calculated and compared with the results obtained by the manual detection. It was found that:

(1) the crack image which was processed by digital image processing method was clear and distinguished significantly from the background;

(2) the crack results obtained by the method proposed in this study had small errors with the manual detection results, and the average errors of the length and width were $0.024 \mathrm{~mm}$ and $0.021 \mathrm{~mm}$ respectively;

The experimental results verified that the method was reliable in crack monitoring, which is conductive to improving the crack monitoring efficiency and scientifically analyzing crack structure and moreover makes some contributions to the safety monitoring and restoration of buildings.

\section{ACKNOWLEDGEMENT}

7 his study is supported by research on the construction of the curriculum system of innovation and entrepreneurship in Colleges and Universities under the background of "Internet +" under grant number jg2018097.

\section{REFERENCES}

[1] Prasanna, P., Dana, K. J., Gucunski, N., et al. (2016). Parvardeh H. Automated Crack Detection on Concrete Bridges, IEEE T. Autom. Sci. Eng., 13(2), pp. 591-599. DOI: 10.109/TASE.2014.2354314.

[2] Riyadi, S., Sugiarto, A., Putra, S. A. and Setiawan, 1N. A. (2015). Analysis of Digital Image Using Pyramidal Gaussian Method to Detect Pavement Crack, Adv. Sci. Lett., 21(11), pp. 3565-3568. DOI: 10.1166/asl.2015.6579.

[3] Kim, H., Lee, J., Ahn, E., et al. (2017). Concrete Crack Identification Using a UAV Incorporating Hybrid Image Processing, Sensors, 17(9), pp. 2052. DOI: 10.3390/s17092052. 
[4] Hoang, N. D. and Nguyen, Q. L. (2018). A novel method for asphalt pavement crack classification based on image processing and machine learning, Engineering with Computers, Accepted, In Press (Part 1), pp. 1-12. DOI: $10.1007 / \mathrm{s} 00366-018-0611-9$.

[5] Lu, C., Yu, J. and Leung, C. K. Y. (2016). An improved image processing method for assessing multiple cracking development in strain hardening cementitious composites (SHCC), Cement Concrete Comp., 74, pp. 191-200. DOI: 10.1016/j.cemconcomp.2016.10.005.

[6] Felli, F., Brotzu, A., Pilone, D. and Vendittozzi, C. (2014). Use of FBG sensors for monitoring cracks of the equestrian statue of Bartolomeo Colleoni in Venice, Frat. Integr. Strut., 8(30), pp. 48-54. DOI: 10.3221/IGF-ESIS.30.07.

[7] Ren, Z. X., Bi, J. H., Xie, L. and Zhang, K. F. (2014). Quantitative detection of wood surface defects by image segmentation method, Chin. J. Liq. Cryst. Displays, 29(5), pp. 785-792. DOI: 10.3788/YJYXS20142905.0785.

[8] Lai, Y. R., Tsai, P. C., Yao, C. Y. and Ruan, S. J. (2017). Improved local histogram equalization with gradient-based weighting process for edge preservation, Multimed. Tools Appl. 76(1), pp. 1-29. DOI: 10.1007/s11042-015-3147-7.

[9] Aranda, L. A., Reviriego, P. and Maestro, J. A. (2017). Error Detection Technique for a Median Filter, IEEE T. Nucl. Sci., pp. 1-1. DOI: $10.1109 /$ TNS.2017.2666843.

[10] Prasad, D. S. and Reddy, B. S. (2017). Digital image processing techniques for estimating power released from the corona discharges, IEEE T. Dielect. El. In., 24(1), pp. 75-82. DOI: 10.1109/TDEI.2016.005896

[11] Robertson, S., Azizpour, H., Smith, K. and Hartman, J. (2018). Digital image analysis in breast pathology-from image processing techniques to artificial intelligence, Transl. Res. J. Lab. Clin. Med., 194, pp. 19. DOI: 10.1016/j.trsl.2017.10.010.

[12] Scholz, M., Konen, W., Tombrock, S., et al. (2015). Development of an endoscopic navigation system based on digital image processing, Comput. Aided Surg., 3(3), pp. 134-143. DOI: 10.1002/(SICI)1097-0150(1998)3:33.0.CO;2-T

[13] Zhang, J.J. and Meng, X.Q. (2015). Defect detection of wire rope for oil well based on adaptive angle, Frat. Integr. Strut., 9(34). DOI: 10.3221/IGF-ESIS.34.65.

[14] Boschetto, A., Bottini, L., Campana, F., Consorti, L. and Pilone, D. (2013). Investigation via morphological analysis of aluminium foams produced by replication casting, Frat. Integr. Strut., 7(26), pp. 01. doi: 10.3221/IGF-ESIS.26.01.

[15] Ambadiyil, S., Prakash, D., Sheeja, M. K. and Pillai, V. P. M. (2017). Secure Storage and Analysis of Fingerprints for Criminal Investigation using Holographic Techniques, Mater. Today Proc., 4(2), pp. 4389-4395.

DOI: 10.1016/j.matpr.2017.04.010.

[16] Pizurica, A., Platisa, L., Ruzic, T., et al. (2015). Digital Image Processing of The Ghent Altarpiece: Supporting the painting's study and conservation treatment, IEEE Signal Proc. Mag., 32(4), pp. 112-122.

DOI: 10.1109/MSP.2015.2411753. 